\title{
Hybrid Technique for Spectrum Sharing in Cognitive Radio Networks for the Internet of Things
}

\author{
Nasrin Sadat Zarif \\ Department of Electrical and \\ Computer Engineering \\ Faculty of 17 Shahrivar, Alborz \\ Branch, Technical and Vocational \\ university (TVU) \\ Alborz, Iran
}

\author{
Abolfazl Qiyasi Moghadam \\ Department of Electrical and \\ Computer Engineering \\ Faculty of Shahid Shamsipour, \\ Tehran Branch \\ Technical and Vocational university \\ (TVU), Tehran, Iran
}

\author{
Mehdi Imani \\ Department of Electrical and \\ Computer Engineering \\ Faculty of Shahid Beheshti, Alborz \\ Branch, \\ Technical and Vocational university \\ (TVU), Alborz, Iran
}

\begin{abstract}
Cognitive Radio (CR) has been introduced and developed for wireless networks. CR is playing an important role in wireless spectrum and with the help of CR, senders can choose the best spectrum for communication. Spectrum Sharing is one of the components of $\mathrm{CR}$ architecture which is responsible for distributing the spectrum among users according to their needs. In addition, it is one of the key challenges to improve the wireless network performance. How to access the spectrum is an important issue in spectrum sharing. Primary Users (PUs) and Secondary Users (SUs) access the spectrum bands based on the overlay and underlay spectrum sharing techniques but SUs are limited in both overlay and underlay. After analyzing the existing mechanisms in this paper, we provide a new mechanism to improve SUs accessing the spectrum. Our mechanism works based on SUs' location and the distance between sender and receiver. The proposed mechanism in this paper shows that SUs can own the spectrum permanently without any interferences with PUs. Also, there is no need for SUs to change or leave the spectrum when PUs return. The proposed method is very useful and efficient due to increasing the performance of CR in different wireless networks. Our proposed method can be considered as a step towards the development of IoT and support the future devices in terms of spectrum access. Our proposed mechanism requires no additional hardware, therefore, its implementation is costless and simple.
\end{abstract}

\section{Keywords}

Cognitive Radio Networks; Spectrum Sharing; IoT; underlay Spectrum Sharing; Overlay Spectrum Sharing

\section{INTRODUCTION}

Joseph Mitola introduced Cognitive Radio (CR) for the first time in 1999. He worked on presenting smart radios that have abilities like collecting information from environment, planning and deciding independently $[1,2,3]$. Simon Haykin expressed a smarter definition that $\mathrm{CR}$ is a wireless communication system capable of being aware of environment and be able to learn and adopt [4]. Generally, CR is a reliable communication (every time and everywhere) and effective spectrum utilization. CR concepts, based on mentioned definitions, are abilities to recognize, selfawareness, intelligence, learn, adopt, reliability and efficiency.

Cognitive Radio technology can be recognized as one of the most used technologies in the Internet of Things (IoT) [5-9] and integration of this technology with future architecture and services to support IoT is expected. Combining these technologies namely CRIoT can improve both efficiency and reliability in wireless networks. Cognitive Radio capabilities such as spectrum sensing, spectrum sharing, dynamic learning form environment and self-adeptness allow us to have more efficient communication. It should be noted that the traditional spectrum sharing techniques in wireless networks do not respond to the IoT anymore due to the increasing number of devices. Thus CR will overcome this problem with spectrum sharing capability as well as developing and supporting it for future objects to have no problem with spectrum access [10].

CR technology addresses the existing problems in wireless networks which are limited by spectrum bands (e.g. using the frequency band and improper spectrum usage issues) [11]. The base concept of this technology is to detect a free channel for better usage. The number of channels depends on some conditions that will be considered in the following sections. There are two kind of users in CR: Primary or licensed User (PU) and Secondary or unlicensed User (SU). PUs are users that have more priority or legal rights to use spectrum band and on the other hand, SUs are users that have no permission to use spectrum or have less priority than PUs. PUs also must use the spectrum without any interference with PUs.

Several methods have been proposed to determine the distance between nodes such as: Received Signal Strength (RSS), Time of Arrival (ToA), Time Differential of Arrival (TDoA) and Angle of Arrival (AoA) [12-16]. Each of them has its pros and cons. In RSS method we need Received Signal Strength Indicator (RSSI) in receiver in order to measure the received signal. Another problem of this method is signal path loss, because this method is based on signal strength and environment also affects signal thus it provides incorrect and unknown results. TDoA is obtained by differential received phases or time differential measurement which presents good accuracy as well as no need to have calibration with the target node but a tight synchronization among all anchors is required. The AoA calculates the input signal angle with the help of TDoA which is mostly used to obtain the geolocation of the nodes (e.g. cell phone). This method has low accuracy and requires costly hardware while the ToA obtains the location based on the arrival time of the signal to the receiver. ToA has high accuracy but the problem is that it requires calibration with the target node. In this paper, we use ToA to obtain the location of nodes. We prove that this method is more efficient than other methods and improves the performance of wireless network in positioning between nodes. The rest of this paper is organized as follows: 
section 2 analyses the different methods in spectrum sharing and node positioning, a background about spectrum sharing in cognitive radio will be presented in section 3 , we describe and prove our proposed mechanism along with obtaining the distance between nodes in order to use the spectrum in hybrid mode in section 4, and finally, we conclude the results of this paper in section 5 .

\section{RELATED WORKS}

Cognitive radio techniques are used for reducing alleviative inefficient spectrum. Spectrum sharing based on access techniques in CR are classified into two types of Overlay and Underlay [17-27] which will be discussed in next section. Channel access technique for SUs depends on the usage of PUs. Both SU and PU can use the spectrum in cooperative way if SU causes no interference with PU [18].

The SUs in underlay technique can adjust their transmission speed to access the channel in an aggressive way because in overlay technique (when the channel is free), SUs access to the channel in an opportunistic way. So overlay technique is more practical than underlay technique since the overlay does not need to be aware of the user presence or knowing the channel interference rate. There are many studies on cognitive radio networks that use hybrid methods and queuing theory [28-30]. Authors in [31] applied queuing theory in order to increase efficiency and maximizing transmission rate for SU in the hybrid networks. In this method the channel capacity is measured and the transmission speed of SU is determined in a way that prevents interference with PU. Therefore, it guarantees the QoS of PUs. Presented protocols in [29,30] treat SUs in the same approach and analyze them based on queuing theory. In hybrid systems, SU is able to change the access technique to underlay thus the spectrum usage increases for SU. The SU is allowed to send packages to the destination even in the presence of PUs in channel. The transmission power of secondary transmitter must be adjusted below the interference threshold in underlay mode so this method is useful for SU when the distance between SU and PU is short. But long distance decreases the transmission power and causes performance destruction for PU so it will not be an efficient method.

Hybrid techniques presented for the first time in Ad Hoc Network, namely Hybrid Cognitive Radio Ad Hoc Network (H-CRAHN) [32]. The transmission power of a node in HCRAHN is varies and it is based on the distance between the SU and its neighboring PU. In addition, the connection in the H-CRAHN networks depends on the number of active PUs [33].

Authors in [34] changed the SU access mode in dynamic way to increase their use of spectrum. In this method the SU transmission mode depends on PUs activity. SUs adjust their transmission power to a specific switching rate when PU activity is detected. Authors in [34] presented ways to optimize the switching rate for balancing the SU spectrum access.

The access strategy with the ability of energy harvesting is presented in [35]. SU is equipped with a rechargeable battery and uses both overlay and underlay techniques to increase the maximum power. This method focuses on the energy harvesting from the environmental resources or PU channel.

SUs use a common relay and compete together based on transmission power through relay [36]. Each node in cognitive radio network must adopt and change the sending and receiving parameters based on the environment conditions.
The connection between nodes in CR becomes possible by using the intermediate nodes called relay when there is no direct communication between them. Selecting a better relay from many nodes is difficult in limited time and has a direct impact on network throughput. In addition, it is impossible to check all nodes to select the best one. We discuss about spectrum sharing techniques in next section.

\section{SPECTRUM SHARING BACKGROUND}

The distribution of spectrum among all CR users based on their usage is spectrum sharing. Accessing the channel in a way to prevent from PU destruction are spectrum sharing responsibilities. Sharing techniques can be categorized in 4 groups: based on utilization of spectrum, based on network architecture, based on allocation behavior and based on access technology. This paper focuses on spectrum sharing based on access technology which will be discussed in detail in the following.

In general, Spectrum Sharing based on access technology is divided into two categories: Overlay access technique and Underlay access technique.

In Overlay access technique, a specific part of spectrum is available for the PU and it is allowed to use it with no limits. On the other hand, SU uses the spectrum when it is free but if PU returns to the spectrum, SU has to leave the spectrum or change to another free channel. Therefore, the most important responsibility of $\mathrm{SU}$ is to sense the spectrum or detect the spectrum holes (holes are part of spectrum band assigned to the PU but they have not been used at a specific time and place). Spectrum Sensing is the ability of SU to sense the spectrum based on common detection methods like: energy detector, coinciding filter and etc. Cooperative spectrum sensing is suggested in order to increase the overlay performance which SUs share their sensed data [17]. In cooperative sensing, SUs may share incorrect sensed data and cause wrong decisions. SUs are facing some challenges in this technique which are:

- Select parts of the spectrum that is accessible.

- Select the best available channel.

- Cooperate with other users to access to the channel.

- Leaving the channel when PU appears.

When SU is sending data and PU returns to the channel, SU must switch to another free channel. Regardless to the problems that occur by frequent stops for sending and receiving, successive switching takes time and reduces the efficiency of SU.

In underlay access technique, PUs and SUs can use the spectrum simultaneously. If SU adjusts its transmission rate to prevent the interference with $\mathrm{PU}$, therefore, the spectrum efficiency increases. Determining the transmission power with least interference level as well as using the channel capacity in order to be efficient for both PUs and SUs are most important challenging issues in this technique.

Each of the overlay or underlay methods has their own weaknesses. Therefore, a new combination of overlay/underlay is suggested for taking a more efficiency from spectrum which will be considered in the next section. 


\section{PROPOSED MECHANISM}

As mentioned already, and according to the advantages of two above techniques, the combination of them namely hybrid mechanism is more useful than previous methods. The proposed mechanism that will be discussed in the following is taking advantage from hybrid method.

We assume that there are two groups of users that located in a point. The number of PUs is equal to authorized channels but the number of SUs may be different. Users in the first group are connected to a communication center but in the second group they are not connected to any center and also they have no channel. Users in the first group declare their status by $\mathrm{ON}$ and OFF and notify the center. Figure 1, shows that members of the second group which need a free channel to transmit data but they do not have any spectrum. Also in our proposed mechanism, the connection occurs when the SU can detect the nearest inactive PU.

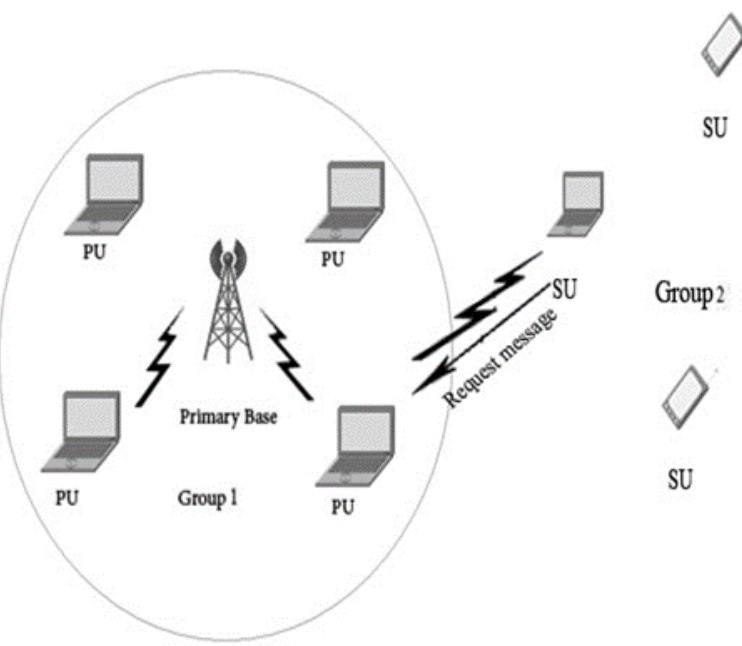

Fig 1: Neighboring node detection by $\mathrm{SU}$ in proposed mechanism.

The SU begins to propagate a message in environment in order to detect the nearest neighbor. This message is a small packet which sender transmits and waits for the response. Figure 1, shows the transmitted message. All PUs receive this message and each one must respond to it in any ON or OFF status. PUs send their status as a response message to the sender. SU receives the responses and selects the nearest inactive PU based on OFF status and distance calculation.

In this paper we assume that PU can be a sender or a receiver and each PU determines its status with ON or OFF. PU is active when it is ON (sending and receiving) and OFF means it does not use its spectrum. In this paper, the inactivity probability of PU considered based on Poisson distribution which formulated as following [33]:

$$
f(x, \lambda)=\frac{\lambda_{a c t}^{x}}{x !} e^{-\lambda_{a c t}}
$$

Where $e$ is natural logarithm base (2.71282), $x$ is OFF PUs and $\lambda_{\text {act }}$ is a positive number equal to mathematical hope of PUs in OFF status. Since in the proposed mechanism, PU will be selected based on shortest distance and using the above formula proves that there are always be some inactive PUs. Therefore, it can be concluded that SUs always can access the channel through their neighbors (SU is always able to find the nearest inactive PU based on shortest distance and takes the spectrum of that PU). In proposed method, we assume that there is line of sight $(\operatorname{LoS})$ between sender and receiver. Moreover, we use a method based on Time of Arrival (ToA) to calculate the distance between sender and receiver. We calculate the distance between sender and receiver based on packet propagation time (which obtained from sending and receiving of message) according to round trip time [12]. The relative distance $R_{t}$ calculate in meter by following equation:

$$
R_{t}=c \times t
$$

Above equation estimates the distance by using time which $c=3 \times 10^{8}$ is the speed of packet (in speed of light) and $t$ is to measure the sending time.

We use operations such as calibration of sender and receiver and delays calculation between them to obtain the exact distance. The Internal Delays $I_{d}$ of sender and receiver must be calculated in order to calibrate them [12]. Thus, we define a simple calibration operation which is described as follows.

Internal delays $I_{d}$ are processing delay $d_{\text {proc }}$, queuing delay $d_{\text {quee }}$, transmission delay $d_{\text {trans }}$ and propagation delay $d_{\text {prop }}$ and each one is obtained by different calculations in a way that processing and queuing delays are near zero due to the size and number of packet or even transmission delay has a constant value. On the other hand, $d_{\text {prop }}$ must be calculated based on the result of formula (2) which is the $R_{t}$ and the speed of packet in wireless space $c=3 \times 10^{8}$. Thus, our relation for propagation delay is $R_{t} / c$. The answer of $I_{d}$ is the sum of all these mentioned delays which equation (3) refers to it.

$$
I_{d}=d_{\text {proc }}+d_{\text {quee }}+d_{\text {trans }}+d_{\text {prop }}
$$

Finally, the exact distance between the transmitter and receiver obtained by using calibration that includes delays calculation. Equation (4) shows the distance calculation.

$$
D=c \times\left(R_{t}-I_{d}\right)
$$

Where $D$ is the distance between sender and receiver, $c$ is speed of light, $R_{t}$ is relative distance and $I_{d}$ is internal delays. Free Space Path Loss (FSPL) estimation is possible by having the distance. As we mentioned before, there is line of sight between sender and receiver and the distance is short. Therefore, FSPL is possible to be estimated. To estimate FSPL, we need some parameters like the distance between sender and receiver $D$, strength of each one as $P_{s}$ and $P_{r}$ and signal length $\lambda$ which is possible to use $(4 \times \pi \times D)$ as sender power and $\lambda=\frac{C}{F}$ as receiver power and fetching the distance $D$ from the equation (4). Thus FSPL estimation is as follows:

$$
\frac{P_{s}}{P_{r}}=\frac{(4 \times \pi \times D)}{\lambda}=\frac{(4 \times \pi \times D) \times f}{c}
$$

If we want to calculate FSPL based on decibel (db) then we need to use logarithmic equations which the equation (6) describes it:

$$
F S P L_{d b}=20 \log \frac{(4 \times \pi \times D) \times f}{c}
$$

By assuming line of sight and short distance in wireless network, the loss of signal in the path becomes less. As we mentioned and considered before, some PUs always are inactive. So the number of detected distances are based on the $n$ neighboring nodes when their response packet arrives to the sender (all the responses do not arrive at the same time due to their distance differences and only the OFF PUs will be calculated). Once the distance between each sender and 
receivers is calculated, sender must determine that which one is proper for communication based on shortest distance.

$\mathrm{SU}$ is able to use the channel in hybrid which is changing to underlay mode when PU returns. Distance is very important in this mode. SUs can communicate with the users in first group when the environment is surrounded by inactive users. It is easy to exchange information in these circumstances. Thus, the hybrid mode will be efficient. Figure 2, shows that how $\mathrm{SU}$ is able to reach the spectrum in hybrid mode by owning the proposed mechanism.

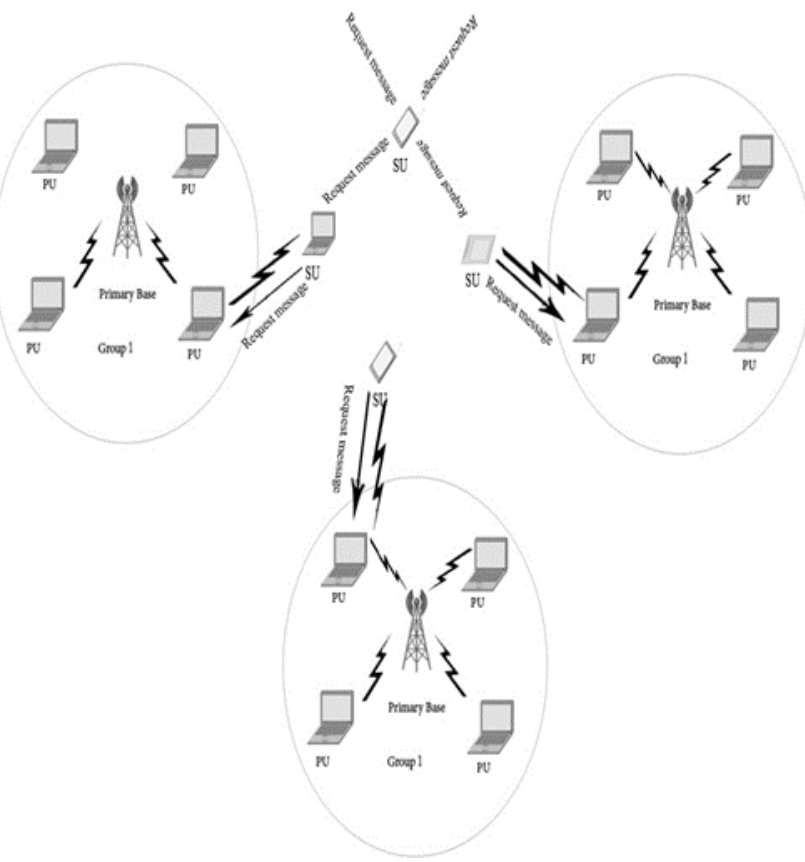

Fig 2: Hybrid communication between $\mathrm{PU}$ and $\mathrm{SU}$ in proposed mechanism.

Although the efficiency and information exchanging trend for $\mathrm{SU}$ is increased but also the switching operation and frequent stops in overlay mode are decreased in proposed method. Using underlay technique permanently based on distance of users includes some advantages like improving the QoS of spectrum and increasing the performance of SU. The proposed method, also causes reducing costs and saving more energy. Improving the spectrum efficiency is a great step in improving the performance and application of cognitive radio in IoT.

Problems in signal propagation are: path loss which has been solved, multipath propagation that has a direct relation with path loss, and distance. But in proposed method, multipath propagation decreases because of short distance between the sender and receiver so the path loss becomes less either.

\section{CONCLUSION}

The purpose of this paper was to present a new mechanism to overcome the inefficient spectrum. In this paper we proposed a mechanism to increase the spectrum efficiency along with preventing interference of SU with PU based on hybrid systems. The study shows that by determining the distance between the PUs and SUs, the efficiency rate of the underlay technique can be increased. The proposed mechanism indicates the application of ToA in order to estimate the relative distance between the sender and receiver. In addition, we suggest the estimation of the signal loss in the path for the sender and the receiver because knowing the signal loss is one of the important issues in signal propagation which this paper refers to it. More studies are required in this field. We claim that the proposed mechanism increases the efficiency and quality of service in spectrum sharing. For future work, we are studying to use quorum-based methods in cognitive radio networks which introduced in $[8,37,38]$.

Another aspect that can be considered for future work is designing an accurate and a precise algorithm for detecting the spectrum hole in high frequency channel, since the conventional spectrum sensing algorithms have problems in this case. Finally, researchers should develop a cognitive radio technology in the field of 5G and should create optimal spectrum sensing/access mechanisms which meet future networks requirements to maximize the efficiency of spectrum utilization.

\section{REFERENCES}

[1] J. Mitola III, Joseph and others, Cognitive radio: making software radios more personal, Personal Communications, IEEE 6 (4) (1999) 13-18.

[2] J. Mitola III, Cognitive radio for flexible mobile multimedia communications, in: Mobile Multimedia Communications, 1999. (MoMuC'99) 1999 IEEE International Workshop on, IEEE, 1999, pp. 3-10.

[3] J. Mitola, et al., Cognitive radio: An integrated agent architecture for software defined radio, Doctor of Technology, Royal Inst. Technol. (KTH), Stockholm, Sweden (2000) 271-350.

[4] S. Haykin, Cognitive radio: brain-empowered wireless communications, Selected Areas in Communications, IEEE Journal on 23 (2) (2005) 201-220.

[5] L. Atzori, A. Iera and G. Morabito, "The internet of things: A survey," Computer Networks, vol. 54, pp. 2787-2805, 2010.

[6] R. Khan, S. U. Khan, R. Zaheer and S. Khan, "Future internet: The internet of things architecture, possible applications and key challenges," in Frontiers of Information Technology (FIT), 2012 10th International Conference On, 2012, pp. 257-260.

[7] J. Gubbi, R. Buyya, S. Marusic and M. Palaniswami, "Internet of Things (IoT): A vision, architectural elements, and future directions," Future Generation Comput. Syst., vol. 29, pp. 1645-1660, 2013.

[8] M. Imani, M. Joudaki, H. R. Arabnia, and N. Mazhari, "A survey on asynchronous quorum-based power saving protocols in multi-hop networks," Journal of Information Processing Systems, vol. 13, no. 6, pp. 1436 - 1458, 2017. https://doi.org/10.3745/JIPS.03.0082

[9] M. Imani, A. Qiyasi Moghadam, N. Zarif, H. R. Arabnia, "A Comprehensive Survey on Addressing Methods in the Internet of Things", 2018, Unpublished.

[10] A. Ali. Khan, M. Husain. Rehmani, A. Rachedi, Cognitive-Radio-Based Internet of Things: Applications, Architectures, Spectrum Related Functionalities, and Future Research Directions, in proceeding of IEEE Wireless Communications, Vol. 24, pp. 17-25, June 2017.

[11] Faisal Fayyaz Qureshia, Rahat Iqbalb, Muhammad Nabeel Asghar" Energy efficient wireless communication technique based on Cognitive Radio for Internet of Things", 2017. 
[12] N. Patwari, J.N. Ash, S. Kyperountas, A.O. Hero, R.L. Moses, N.S. Correal, Locating the nodes: cooperative localization in wireless sensor networks, in proceedings of IEEE Signal Processing Magazine, Vol. 22, Issue. 4, pp. 54-69, July 2005.

[13] Han G, Xu H, Duong TQ, Jiang J, Hara T. Localization algorithms of wireless sensor networks: a survey. Telecommunication Systems. pp, 1-8, 2013 Apr

[14] S. Halder, A. Ghosal, 2016. A survey on mobilityassisted localization techniques in wireless sensor networks. Journal of Network and Computer Applications, Vol. 60, pp. 82-94, January 2016.

[15] K. Jeril, V. Amruth, N. Swathy Nandhini. "A survey on localization of wireless sensor nodes." In Information Communication and Embedded Systems (ICICES), 2014 International Conference on, pp. 1-6. IEEE, 2014.

[16] Piccinni, G., G. Avitabile, and G. Coviello. "A novel distance measurement technique for indoor positioning systems based on Zadoff-Chu Sequences." In New Circuits and Systems Conference (NEWCAS), 2017 15th IEEE International, pp. 337-340. IEEE, 2017.

[17] Yifeng, C.; Huazhong, U. "Optimal Data Fusion of Collaborative Spectrum Sensing under Attack in CognitiveRadio Networks Network"; IEEE Net. 2014, 1, 17-23.

[18] Federal Communications Commission, Spectrum policy task force report, (ETDocket No.02-135), Nov. 2002.

[19] A. Ghasemi, and E. S. Sousa, "Fundamental limits of spectrum sharing in fading environments", IEEE Trans. Wireless Commun, vol. 6, no. 2, pp. 649-658, Feb. 2007.

[20] T. W. Ban, W. Choi, B. C. Jung, and D. K. Sung, "Multiuser diversity in a spectrum sharing system", IEEE Trans. Wireless Commun, vol. 8, no. 1, pp. 102-106, Jan. 2009.

[21] T. W. Ban, D. K. Sung, B. C. Jung, and W. Choi, "Capacity analysis of an opportunistic scheduling system in a spectrum sharing environment," in Proc. IEEE Globecom, Nov. 2008

[22] R. Zhang, and Y.-C. Liang, "Exploiting multi-antennas for opportunistic spectrum sharing in cognitive radio networks," IEEE J. Select. Topics Signal Processing, vol. 2, no. 1, pp. 88-102, Feb. 2008.

[23] J. Mitola and G. Q. Maguire, "Cognitive Radios: Making Software Radios More Personal," IEEE Personal Communications, vol. 6, no. 4, pp. 1318, Aug. 1999.

[24] S. Haykin, "Cognitive Radio: Brain-Empowered Wireless Communications," IEEE J. Select. Areas Commun., vol. 23, no. 2, pp. 201-220, Feb. 2005.

[25] S. Srinivasa, and S. A. Jafar, "How much spectrum sharing is optimal in cognitive radio networks, IEEE Trans. Wireless Commun., vol. 7, no. 10, pp. 4010-4017, Oct. 2008.

[26] R. Etkin, A. Parekh, and D. Tse, "Spectrum sharing for unlicensed bands," IEEE J. Select. Areas Commun., vol. 25, no. 3, pp. 517-528, Apr. 2007.
[27] Y. Xing, R. Chandramouli, S. Mangold, and S. Shankar $\mathrm{N}$, "Dynamic spectrum access in open spectrum wireless networks," IEEE J. Select. Areas Commun, vol. 24, no. 3, pp. 626-637, Mar. 2006

[28] O. Simeone, Y. Bar-Ness, and U. Spagnolini, "Stable throughput of cognitive radios with and without relaying capability," IEEE Trans. Commun., vol. 55, no. 12, pp. 2351-2360, Dec. 2007.

[29] I. Krikidis, J. N. Laneman, J. S. Thompson and S. Mclaughlin, "Protocol design and throughput analysis for multi-user cognitive cooperative systems," IEEE Trans. Wireless Commun., vol. 8, no. 9, pp. 4740-4751, Sep. 2009.

[30] J. Gambini, O. Simeone, Y. Bar-Ness U. Spagnolini and T. Yu, "Packetwise vertical handover for unlicensed multi-standard spectrum access with cognitive radios," IEEE Trans. Wireless Commun., vol. 7, no. 12, pp. 5172 5176, Dec. 2008

[31] Jinhyung Oh, Wan Choi, A Hybrid Cognitive Radio System: A Combination of Underlay and Overlay Approaches ,"11573265, Sept. 2010.

[32] S. Senthuran, A. Anpalagan, and O. Das, "Throughput Analysis of Opportunistic Access Strategies in Hybrid Underlay/Overlay Cognitive Radio Networks," IEEE Transactions on Wireless Communications, vol. 11, no 6, pp. 2024-2035, June 2012.

[33] Nhu Tri Do, Le The Dung, Beongku An, Sang-Yep Nam "Connectivity of Hybrid Overlay/Underlay Cognitive Radio Ad Hoc Networks" 16285184, Jan. 2016.

[34] Song, H., Hong, J.-P., \& Choi, W. (2013). On the optimal switching probability for a hybrid cognitive radio system. IEEE Transactions on Wireless Communications, 12, 1594-1605.

[35] Usman, M., \& Koo, I. (2014). Access strategy for hybrid underlay-overlay cognitive radios with energy harvesting. IEEE Sensors Journal, 14, 3164-3173.

[36] Junni Zou, Hongkai Ziong, Dawei Wang, Chang Wen Chen, "optimal power allocation for Hybrid overlay/underlay spectrum sharing in multiband cognitive radio networks", IEEE Transaction on vehicular technology, VOL. 62, NO.4, May 2013.

[37] M. Imani, M. Dehghan "S-Grid: A New Quorum-based Power Saving Protocol to Maximize Neighbor Sensibility.", Proceedings of the IEEE 25th Iranian Conference on Electrical Engineering (ICEE), Tehran, Iran, pp 2134-2139, 2017.

[38] M. Imani, O. Noshiri, M. Joudaki, M. Pouryani, M Dehghan, "Adaptive S-Grid: A New Adaptive Quorumbased Power Saving Protocol for Multi-Hop Ad Hoc Networks.", Proceedings of the IEEE $4^{\text {th }}$ international conference on Knowledge-Based Engineering and Innovation (KBEI). Tehran. Iran. 2017. 